\title{
Asymptotic Behavior of Solutions of the Dirac System with an Integrable Potential
}

\author{
Łukasz Rzepnicki®
}

\begin{abstract}
We consider the Dirac system on the interval $[0,1]$ with a spectral parameter $\mu \in \mathbb{C}$ and a complex-valued potential with entries from $L_{p}[0,1]$, where $1 \leq p$. We study the asymptotic behavior of its solutions in a strip $|\operatorname{Im} \mu| \leq d$ for $\mu \rightarrow \infty$. These results allow us to obtain sharp asymptotic formulas for eigenvalues and eigenfunctions of Sturm-Liouville operators associated with the aforementioned Dirac system.
\end{abstract}

Mathematics Subject Classification. Primary 34L20, Secondary 34E05.

Keywords. Dirac system, Spectral problem, Integrable potential, SturmLiouville operator.

\section{Introduction}

Consider a Cauchy problem

$$
D^{\prime}(x)+J(x) D(x)=A_{\mu} D(x), \quad D(0)=I,
$$

where $x \in[0,1], A_{\mu}=i \mu J_{0}$, and

$$
J_{0}=\left[\begin{array}{cc}
1 & 0 \\
0 & -1
\end{array}\right], \quad J(x)=\left[\begin{array}{cc}
0 & \sigma_{1}(x) \\
\sigma_{2}(x) & 0
\end{array}\right], \quad I:=\left[\begin{array}{ll}
1 & 0 \\
0 & 1
\end{array}\right],
$$

$\mu \in \mathbb{C}$ is a spectral parameter, and $\sigma_{j} \in L_{p}[0,1], j=1,2$ are complex-valued functions where $1 \leq p<2$. We study the asymptotic behavior of its solutions $D(x)=D(x, \mu)$ with respect to $\mu$ from a horizontal strip

$$
P_{d}:=\{\mu \in \mathbb{C}:|\operatorname{Im} \mu| \leq d\} .
$$

and $\mu \rightarrow \infty$.

The solution of (1.1) is a matrix $D$ with entries from the space of absolutely continuous functions on $[0,1]$ (i.e. from the Sobolev space $W_{1}^{1}[0,1]$ ) satisfying $(1.1)$ for a.e. $x \in[0,1]$. In our case, this definition together with the equation imply that $D$ has entries from $W_{p}^{1}[0,1]$. 
This article is an addendum to the paper [8], where the problem (1.1) was analyzed for $\sigma_{j} \in L_{2}[0,1], j=1,2$. In that text one can find a background on Dirac systems and their connection with Sturm-Liouville problems.

Here, we use all advantages of the method from [8] to obtain sharp asymptotic formulas for $D$ and consequently for solutions of spectral problems associated with (1.1). In the case when $\sigma_{j} \in L_{p}[0,1], j=1,2, p>2$ one can use the results from [8] due to the obvious embedding between $L_{p}[0,1]$ spaces. Thus, in this text we restrict our reasoning only to $1 \leq p<2$.

We are interested in the following spectral problem:

$$
Y^{\prime}(x)+J(x) Y(x)=A_{\mu} Y(x), \quad x \in[0,1],
$$

where $Y=\left[y_{1}, y_{2}\right]^{T}$ and

$$
y_{1}(0)=y_{2}(0), \quad y_{1}(1)=y_{2}(1) .
$$

Conditions (1.4) are an example of strongly regular boundary conditions. The Dirac-type systems or equation (1.3) with a general formulation of regular or strongly regular conditions have been studied for many years and by different methods.

The classical results can be found in [4], where G. D. Birkhoff and R. E. Langer obtained refined asymptotic formulas for general $n \times n$ system of the form

$$
\begin{aligned}
L y & =-i B y^{\prime}+Q(x) y=\lambda y, \\
B & =\operatorname{diag}\left(b_{1}, \ldots, b_{n}\right), \quad y=\operatorname{col}\left(y_{1}, \ldots, y_{n}\right), \quad x \in[0,1],
\end{aligned}
$$

where $Q \in C^{m}[0,1] \times \mathbb{C}^{n \times n}, m \geq 1$. These formulas were used to obtain asymptotic formulas for eigenvalues and eigenfunctions of regular boundary value problem and to prove a pointwise convergence result for spectral decompositions. It is worth to mention that these results were generalized by V. S. Rykhlov in [18] for $Q \in L_{1}[0,1] \times \mathbb{C}^{n \times n}$.

For $2 \times 2$ Dirac systems with potential matrix $Q \in C[0,1] \times \mathbb{C}^{2 \times 2}$ the existence of a triangle transformation operator has been proved for the first time by Gasymov and Levitan (see [9]). Application to asymptotic formulas for solutions and eigenvalues can also be found there in chapter vii. In this case, V. A. Marchenko proved completeness property of regular BVP for Dirac operator in his classical monograph [16] (see exercises in $\S 1.3$ ).

Whereas, for $Q \in L_{1}[0,1] \times \mathbb{C}^{n \times n}$ there is a series of paper $[1,12,14,15]$ where asymptotic formulas for solutions were obtained in a special sectors and has been applied to establish the completeness property of regular and non-regular boundary value problems. In $2 \times 2$ case more refined asymptotic formulas were derived using transformation operators.

The Riesz basis property of the root vectors system of the Dirac operator with strictly regular boundary conditions and summable potential matrix $Q \in L_{1}[0,1] \times \mathbb{C}^{2 \times 2}$ was established by A. A. Lunyov and M. M. Malamud [11], [13] on the one hand, and A. M. Savchuk and A. A. Shkalikov in [25] on the other hand, independently by different methods and at the same time. In both papers basic asymptotic formula for solutions to the Dirac system 
were established in the strip $P_{d}$. This result was used to prove the following asymptotic formula

$$
\mu_{n}=\mu_{n}^{0}+o(1)
$$

for the eigenvalues, which was one of the main ingredients for proving a Riesz basis property.

Furthermore A. A. Lunyov and M. M. Malamud have recently presented a preprint [10] where Lipschitz dependence on $Q$ in $l^{p}$-norms of the sequences of eigenvalues and eigenfunctions of BVPs for Dirac operator was proved on compacts and balls in $L_{p}[0,1] \times \mathbb{C}^{2 \times 2}$.

Moreover, in [25] A. M. Savchuk and A. A. Shkalikov obtained for $p \geq 1$ basic asymptotic formulas for eigenvalues and for fundamental solutions of the Dirac-type system only with the leading term and the remainders expressed by $\gamma$ and $\gamma_{q}$ given by (2.21) and (2.22). They got their results by applying Prüfer's substitution.

The works of A. M. Savchuk and I. V. Sadovnichaya: [19], [21] and [22] may be regarded as articles which building up on the method from $[20,25]$ and its application for $p=1$ to problems from the fields of asymptotics formulas and basis properties (see also [23,24]). Almost all aforementioned works prove or use the same type of results as mentioned before since they deal with the Riesz basis property and very detailed formulas are not needed.

In order to study inverse spectral problems S. Albeverio, R. Hryniv and Y. Mykytyuk in [2] investigated a direct spectral problem for the Dirac system in the form

$$
B Z^{\prime}(x)+Q(x) Z(x)=\mu Z(x), \quad x \in[0,1],
$$

where

$$
B=\left[\begin{array}{cc}
0 & 1 \\
-1 & 0
\end{array}\right], \quad Q(x)=\left[\begin{array}{cc}
q_{1}(x) & q_{2}(x) \\
q_{2}(x) & -q_{1}(x)
\end{array}\right], \quad q_{j} \in L_{p}[0,1], \quad j=1,2,
$$

with $p \geq 1$. They proved also short formulas for fundamental system of solutions, where remainders were expressed in terms of Fourier coefficients of unknown functions from $L_{p}$. Furthermore, for the operators associated with the system (1.6) with two kinds of conditions

$$
z_{j}(1)=z_{2}(0)=0, \quad j=1,2,
$$

they presented basic formulas for eigenvalues with the same type of remainders.

More results concerning different type of problems for the Dirac system may be found in the series of paper of P. Djakov and B. Mityagin: [5], [6] and [7] or D. V. Puyda [17].

The main result of this paper concerning Dirac systems is

Theorem 1.1. Let $1 \leq p \leq 2$ and $1 / p+1 / q=1$. Then for every $d>0$ there exist constants $C_{j}=C_{j}\left(d, \sigma_{1}, \sigma_{2}\right), j=0,1,2$ such that for all $x \in[0,1]$ and $\mu \in P_{d}$, the solutions of (1.1) admit the following representation

$$
D(x, \mu)=e^{x A_{\mu}}+R(x, \mu),
$$


where

$$
\|R(x, \mu)\|_{\mathbb{C}^{2 \times 2}} \leq C_{1}\left(\gamma_{q}(\mu)+\gamma(x, \mu)\right) \leq C_{0}, \quad x \in[0,1] .
$$

Moreover,

$$
D(x, \mu)=e^{x A_{\mu}}+D_{0}(x, \mu)+R_{0}(x, \mu),
$$

where

$$
D_{0}(x, \mu):=\int_{0}^{x} e^{(x-2 t) A_{\mu}}(-J(t)+\tilde{J}(x, t)) d t
$$

and

$$
\left\|R_{0}(x, \mu)\right\|_{\mathbb{C}^{2 \times 2}} \leq C_{2}\left(\gamma_{q}(\mu) \gamma(x, \mu)+\widetilde{\gamma}(\mu)\right), \quad x \in[0,1],
$$

where $\gamma, \gamma_{0}$ and $\widetilde{\gamma}$ are given by (2.21)-(2.23) and $\tilde{J}$ by (2.8).

Whereas, asymptotic representation for solutions of the spectral problem (1.3)-(1.4) are contained in the following theorem.

Theorem 1.2. The eigenvalues of the spectral problem (1.3)-(1.4) lie in a certain strip $P_{d}$ and admit the representation

$$
\mu_{n}=\pi n+\mu_{0, n}+\rho_{n}, \quad n \in \mathbb{Z},
$$

with

$$
\begin{aligned}
\mu_{0, n} & =\frac{1}{2 i} \int_{0}^{1} e^{-2 \pi i n t} \sigma_{1}(t) d t-\frac{1}{2 i} \int_{0}^{1} e^{2 \pi i n t} \sigma_{2}(t) d t \\
& -i \int_{0}^{1} \int_{0}^{t} \sigma_{1}(t) \sigma_{2}(\xi) e^{-2 \pi i n t} e^{2 \pi i n \xi} d \xi d t
\end{aligned}
$$

and for $p=1$ it holds that

$$
\left|\rho_{n}\right|<c \Gamma^{2}(\pi n)
$$

where $\Gamma$ is defined in (2.24), whereas for $1<p<2$ it is true that

$$
\sum_{n \in \mathbb{Z}}\left|\rho_{n}\right|^{q / 2}<\infty .
$$

The result by A. M. Savchuk and A. A. Shkalikov from [25] is equivalent to first thesis (1.8) of Theorem 1.1. Note that the next statement (1.9) is a significant extension of the previous result. Its version for $p=1$ may be found in Remark 2.4. The most general result is the content of Lemma 2.2.

Using our method it is also possible to obtain very detailed formulas for eigenvalues and eigenfunctions. In case of the spectral problem associated with (1.4) the eigenvalues admit the representation (1.10)-(1.11) with remainders satisfying (1.12) and (1.13) for $p=1$ and $1<p<2$ respectively. In literature (for instance in [25]) for $1<p<2$ one may find results which state that eigenvalues are of the form $\pi n+r_{n}$, where $\left(r_{n}\right) \in l_{q}$, and $q$ is conjugated to $p$. Here it is worth to underline that beside the leading term in our asymptotic formulas there occur Fourier coefficients of known functions and the remainder, which belongs to $l_{q / 2}$. Additionally, for $p=1$ we extend known formulas with $\left|r_{n}\right|<c \Gamma(\pi n)$ (where $\Gamma$ is defined in (2.24)) into more detailed one with the remainder satisfying $\left|r_{n}\right|<c \Gamma^{2}(\pi n)$. 
In the same spirit Theorem 3.2 and Corollary 3.3 generalize significantly the results on eigenfunctions from literature.

Our method is applicable not only to the spectral problem (1.3)-(1.4) but it works as well for different cases of strongly regular boundary conditions. Moreover, it may be used to deal with the class of regular boundary conditions (in the sense of Birkhoff).

Results by S. Albeverio, R. Hryniv and Y. Mykytyuk from [2] can be directly derived from our approach with the help of transformation $Z=U Y$, where

$$
U=\left[\begin{array}{cc}
1 & -i \\
-i & 1
\end{array}\right]
$$

It leads to the system (1.1) with $\sigma_{1}=q_{1}+i q_{2}$ and $\sigma_{2}=q_{1}-i q_{2}$ with appropriate conditions. The relation between different formulations of Dirac systems is explained deeper in [8].

We start our presentation with the section concerning asymptotic behavior for solutions of Dirac system. Next, in section 3 we apply these results to the aforementioned spectral problem. For the clarity of exposition some technical results are placed at the end in appendix.

\section{Dirac System and its Solutions}

In this section we study the matrix Cauchy problem (1.1) and the behavior of its solution in a special integral form. The idea of this approach was taken from [16, Ch. 1, §24] and developed in [8]. We follow it here directly for similar operators but in different function spaces.

First, we introduce necessary notation. We use throughout the text a standard symbol $L_{p}[0,1], p \geq 1$ to denote the space of measurable complex functions integrable with $p$-th power with the classical norm

$$
\|f\|_{L_{p}}=\left(\int_{0}^{1}|f(x)|^{p} d x\right)^{1 / p} .
$$

We write $l_{p}, p \geq 1$ for the space of complex sequences summable with $p$-th power and endowed with the norm

$$
\left\|\left(x_{n}\right)\right\|_{p}=\left(\sum_{n \in \mathbb{Z}}\left|x_{n}\right|^{p}\right)^{1 / p} .
$$

$W_{p}^{1}[0,1]$ is a standard Sobolev space with the derivative in $L_{p}[0,1]$.

If $X$ is a Banach space, then $M(X)$ stands for the Banach space of $2 \times 2$ matrices with entries from $X$ and the norm

$$
\|Q\|_{M(X)}:=\sum_{k, j=1}^{2}\left\|Q_{j k}\right\|_{X}, \quad Q=\left[Q_{j k}\right]_{j, k=1}^{2} .
$$

We assume throughout the text that $1 \leq p<2$. Moreover, let $q$ and $p$ be conjugate exponents and $r$ be a number from Young's convolution inequality 
i.e.

$$
\frac{1}{p}+\frac{1}{q}=1 \text { and } r=\frac{p}{2-p}
$$

Let

$$
\Delta:=\left\{(x, t) \in \mathbb{R}^{2}: 0 \leq t \leq x \leq 1\right\}
$$

and

$$
\begin{gathered}
B:=\left\{f:[0,1] \times[0,1] \rightarrow \mathbb{C} \text { a.e. }: \forall_{x \in[0,1]} f(x, \cdot) \in C\left([0,1], L_{r}\right),\right. \\
\operatorname{supp} f \subset \Delta)\} .
\end{gathered}
$$

We equip $B$ with the norm

$$
\|f\|_{B}:=\sup _{x \in[0,1]}\|f(x, \cdot)\|_{L_{r}[0, x]},
$$

so that $B$ is a Banach space. In particular, directly from the definition if $f \in B$, then $f(x, t)=0$ for $0 \leq x<t \leq 1$. This comment allows us to underline the property which will be used in the text i.e. for $f \in B$ there it holds that

$$
\int_{0}^{x} f(x, t) d t=\int_{0}^{1} f(x, t) d t \in C[0,1] .
$$

We will use the series of constants connected with functions $\sigma_{j}, j=1,2$ in our estimations:

$$
a:=\left\|\sigma_{1}\right\|_{L_{1}} \cdot\left\|\sigma_{2}\right\|_{L_{1}}, \quad a_{1}:=\left\|\sigma_{1}\right\|_{L_{1}}+\left\|\sigma_{2}\right\|_{L_{1}}, \quad a_{p}:=\left\|\sigma_{1}\right\|_{L_{p}}+\left\|\sigma_{2}\right\|_{L_{p}} .
$$

Moreover, let

$$
\sigma_{0}(x):=\left|\sigma_{1}(x)\right|+\left|\sigma_{2}(x)\right| \in L_{p}[0,1] .
$$

Now we are ready to establish a first crucial property of the solutions of (1.1). The proof of the following lemma relays on technical results related to certain integral operators, which are placed in appendix.

Lemma 2.1. Let $\sigma_{0} \in L_{p}[0,1], 1 \leq p<2$.

a) The unique solution $D=D(x, \mu)$ of Cauchy problem (1.1) can be represented as

$$
D(x, \mu)=e^{x A_{\mu}}+\int_{0}^{x} e^{(x-2 t) A_{\mu}}[-J(t)+Q(x, t)] d t,
$$

where $Q \in M(B)$ is the unique solution of the integral equation

$$
Q(x, t)=\tilde{J}(x, t)-\int_{0}^{x-t} J(t+\xi) Q(t+\xi, \xi) d \xi
$$

with $\tilde{J} \in M(B)$ given by

$$
\tilde{J}(x, t):=\int_{0}^{x-t} J(t+\xi) J(\xi) d \xi=\int_{t}^{x} J(s) J(s-t) d s, \quad(x, t) \in \Delta .
$$


b) The following estimates hold:

$$
\|Q\|_{M(B)} \leq C_{1}, \quad\|D\|_{M(C[0,1])} \leq C_{2}, \quad \mu \in P_{d}
$$

with certain constants $C_{j}=C_{j}\left(d, \sigma_{1}, \sigma_{2}\right), j=1,2$.

Proof. Note that the uniqueness of solutions follows from general results on Sturm-Liouville equations (for instance [26, Thm. 1.2.1]). We look for solutions of (1.1) in a special form

$$
D(x, \mu)=e^{x A_{\mu}} U(x, \mu), \quad U(0, \mu)=I .
$$

The identity

$$
J(x) e^{x A_{\mu}}=e^{-x A_{\mu}} J(x), \quad \text { a. e. } x \in[0,1]
$$

implies that $U$ satisfies the Cauchy problem

$$
U^{\prime}(x, \mu)+e^{-2 x A_{\mu}} J(x) U(x, \mu)=0, \quad x \in[0,1], \quad U(0, \mu)=I,
$$

which is equivalent to the integral equation

$$
U(x, \mu)=I-\int_{0}^{x} e^{-2 t A_{\mu}} J(t) U(t, \mu) d t, \quad x \in[0,1] .
$$

We will seek for solutions of (2.12) of the form

$$
U(x, \mu)=I+\int_{0}^{x} e^{-2 t A_{\mu}} Q_{0}(x, t) d t,
$$

where $Q_{0} \in M(B)$ does not depend on $\mu$. Inserting (2.13) into (2.12), we obtain

$$
\begin{aligned}
\int_{0}^{x} e^{-2 t A_{\mu}} Q_{0}(x, t) d t & =-\int_{0}^{x} e^{-2 t A_{\mu}} J(t) d t \\
& -\int_{0}^{x} e^{-2 t A_{\mu}} J(t) \int_{0}^{t} e^{-2 s A_{\mu}} Q_{0}(t, s) d s d t .
\end{aligned}
$$

Due to the fact that

$$
J_{0}^{2}=I, \quad J_{0} J(x)+J(x) J_{0}=0, \quad \text { a.e. } x \in[0,1],
$$

we get

$$
\begin{gathered}
\int_{0}^{x} e^{-2 t A_{\mu}} J(t) \int_{0}^{t} e^{-2 s A_{\mu}} Q_{0}(t, s) d s d t=\int_{0}^{x} e^{-2 t A_{\mu}} \int_{0}^{t} e^{2 s A_{\mu}} J(t) Q_{0}(t, s) d s d t \\
=\int_{0}^{x} e^{-2 t A_{\mu}} \int_{0}^{x-t} J(t+\xi) Q_{0}(t+\xi, \xi) d \xi d t
\end{gathered}
$$

thus

$$
\int_{0}^{x} e^{-2 t A_{\mu}} Q_{0}(x, t) d t=-\int_{0}^{x} e^{-2 t A_{\mu}}\left(J(t)+\int_{0}^{x-t} J(t+\xi) Q_{0}(t+\xi, \xi) d \xi\right) d t
$$

for all $x \in[0,1]$. We conclude that $U$ is a solution of (2.12) if and only if $Q_{0} \in M(B)$ is a solution of

$$
Q_{0}(x, t)=-J(t)-\int_{0}^{x-t} J(t+\xi) Q_{0}(t+\xi, \xi) d \xi
$$


Next, setting

$$
Q_{0}(x, t)=-J(t)+Q(x, t), \quad(x, t) \in \Delta,
$$

and using (2.15), we infer that $Q$ satisfies (2.7). For $Q$ the equation (2.7) can be rewritten in an operator form

$$
Q=\tilde{J}+\widetilde{T} Q, \quad \widetilde{T}=-\left[\begin{array}{cc}
0 & T_{\sigma_{1}} \\
T_{\sigma_{2}} & 0
\end{array}\right],
$$

for the operators $T_{\sigma_{1}}$ and $T_{\sigma_{2}}$, defined on $B$ by

$$
\left(T_{\sigma} f\right)(x, t)=\int_{0}^{x-t} \sigma(t+\xi) f(t+\xi, \xi) d \xi=\int_{t}^{x} \sigma(s) f(s, s-t) d s,
$$

where $\sigma \in L_{p}[0,1]$.

Observe that

$$
\tilde{J}(x, t)=\left(\begin{array}{cc}
\tilde{\sigma}_{1}(x, t) & 0 \\
0 & \tilde{\sigma}_{2}(x, t)
\end{array}\right)
$$

where

$$
\tilde{\sigma}_{1}(x, t):=\int_{0}^{x-t} \sigma_{1}(t+\xi) \sigma_{2}(\xi) d \xi, \quad \tilde{\sigma}_{2}(x, t):=\int_{0}^{x-t} \sigma_{2}(t+\xi) \sigma_{1}(\xi) d \xi .
$$

According to Lemma $4.1 \tilde{J} \in M(B)$. What is more, the operators $T_{\sigma}$ are linear and bounded on $B$ due to Lemma 4.3. In particular, we have

$$
\|\widetilde{T} F\|_{M(B)} \leq a_{1}\|F\|_{M(B)}, \quad F \in M(B) .
$$

Next observe that

$$
\widetilde{T}^{2 n}=\left[\begin{array}{cc}
T_{12}^{n} & 0 \\
0 & T_{21}^{n}
\end{array}\right], \quad n \in \mathbb{N},
$$

for bounded linear operators $T_{12}$ and $T_{21}$ on $B$ given by

$$
T_{12}:=T_{\sigma_{1}} T_{\sigma_{2}}, \quad T_{21}:=T_{\sigma_{2}} T_{\sigma_{1}} .
$$

Therefore by (4.4), we derive

$$
\left\|\widetilde{T}^{2 n} F\right\|_{M(B)} \leq \frac{a^{n}}{n !}\|F\|_{M(B)}, \quad F \in M(B),
$$

thus we see that (2.7) has a unique solution $Q \in M(B)$ of the form

$$
Q=\sum_{n=0}^{\infty} \widetilde{T}^{n} \tilde{J}=\sum_{n=0}^{\infty} \widetilde{T}^{2 n}(I+\widetilde{T}) \tilde{J}
$$

and moreover

$$
\|Q\|_{M(B)} \leq\left(1+a_{1}\right) e^{a}\|\tilde{J}\|_{M(B)} .
$$

Then (2.18) and (4.1) yield (2.9).

Note that from (2.3) and (2.6) we get $D \in C[0,1]$. Adding together (2.6) and (2.9), we obtain

$$
\|D\|_{M(C[0,1])} \leq e^{d}\left(1+a_{1}+\|Q(x, t)\|_{M(B)}\right), \quad \mu \in P_{d} .
$$


We now proceed to derivation of asymptotic formulas for $D$ with the use of the previous lemma. In what follows we will use different types of estimates for remainders. For fixed $\sigma_{j} \in L_{p}, p \geq 1, j=1,2$, and $\mu \in \mathbb{C}$ define

$$
\gamma_{q}(\mu):=\sum_{j=1}^{2}\left(\left\|\int_{0}^{x} e^{-2 i \mu t} \sigma_{j}(t) d t\right\|_{L_{q}}+\left\|\int_{0}^{x} e^{2 i \mu t} \sigma_{j}(t) d t\right\|_{L_{q}}\right),
$$

where $1 / q+1 / p=1$. We will need also

$$
\gamma(x, \mu):=\sum_{j=1}^{2}\left(\left|\int_{0}^{x} e^{-2 i \mu t} \sigma_{j}(t) d t\right|+\left|\int_{0}^{x} e^{2 i \mu t} \sigma_{j}(t) d t\right|\right), \quad x \in[0,1]
$$

and

$$
\begin{aligned}
\widetilde{\gamma}(\mu) & :=\int_{0}^{1} \sigma_{0}(s) \gamma^{2}(s, \mu) d s, \\
\Gamma(\mu) & :=\sum_{j=1}^{2}\left(\sup _{x \in[0,1]}\left|\int_{0}^{x} e^{-2 i \mu t} \sigma_{j}(t) d t\right|+\sup _{x \in[0,1]}\left|\int_{0}^{x} e^{2 i \mu t} \sigma_{j}(t) d t\right|\right) .
\end{aligned}
$$

Note that $\Gamma$ is nothing else than $\gamma_{q}$ for $p=1$ and $q=\infty$.

It is easy to see that if $\mu \in P_{d}$ then

$$
\gamma(x, \mu) \leq 2 e^{2 d} a_{1}, \quad\|\gamma(x, \mu)\|_{L_{q}} \leq \gamma_{q}(\mu), \quad \gamma_{q}(\mu) \leq 2 e^{2 d} a_{1}, \quad x \in[0,1],
$$

and

$$
\widetilde{\gamma}(\mu) \leq 4 a_{1}^{3} e^{4 d}, \quad \widetilde{\gamma}(\mu) \leq 2 a_{1} e^{2 d}\left\|\sigma_{0}\right\|_{L_{p}} \gamma_{q}(\mu) .
$$

In the following lemma we will need

$$
N(x, t):=(\tilde{J}+\widetilde{T} \tilde{J})(x, t) \in B .
$$

Observe that the explicit form of $N$ is

$$
N(x, t)=\left(\begin{array}{cc}
\tilde{\sigma}_{1}(x, t) & -\left(T_{\sigma_{1}} \tilde{\sigma}_{2}\right)(x, t) \\
-\left(T_{\sigma_{2}} \tilde{\sigma}_{1}\right)(x, t) & \tilde{\sigma}_{2}(x, t)
\end{array}\right) .
$$

The very basic but crucial result uses mainly the description of some integrals connected with the operator $\widetilde{T}$ and its powers stated in Lemma 4.6.

Lemma 2.2. Let $\sigma_{j} \in L_{p}, 1 \leq p<2$ for $j=1$, 2. If $D(\cdot, \mu)$ is the solution of (1.1) then $D(\cdot, \mu) \in C[0,1]$ and

$$
D(x, \mu)=e^{x A_{\mu}}+D^{(0)}(x, \mu)+D^{(1)}(x, \mu),
$$

where

$$
D^{(0)}(x, \mu)=-\int_{0}^{x} e^{(x-2 t) A_{\mu}} J(t) d t+\int_{0}^{x} e^{(x-2 t) A_{\mu}} N(x, t) d t,
$$

and for all $\mu \in P_{d}$ and $x \in[0,1]$,

$$
\left\|D^{(1)}(x, \mu)\right\|_{M(\mathbb{C})} \leq C \widetilde{\gamma}(\mu),
$$

where $C=C\left(d, \sigma_{1}, \sigma_{2}\right)$. 
Proof. Note that by using (2.6) and (2.18) for $D=D(x, \mu), x \in[0,1], \mu \in P_{d}$, we get

$$
\begin{aligned}
D(x, \mu) & =e^{x A_{\mu}}-\int_{0}^{x} e^{(x-2 t) A_{\mu}} J(t) d t+\int_{0}^{x} e^{(x-2 t) A_{\mu}} \tilde{J}(x, t) d t \\
& +\int_{0}^{x} e^{(x-2 t) A_{\mu}}(\widetilde{T} \tilde{J})(x, t) d t+D^{(1)}(x, \mu)
\end{aligned}
$$

where

$$
D^{(1)}(x, \mu)=\int_{0}^{x} e^{(x-2 t) A_{\mu}} \sum_{n=2}^{\infty}\left(\widetilde{T}^{n} \tilde{J}\right)(x, t) d t .
$$

Using (2.30) and the inequality (4.12) proved in appendix, we show that

$$
\begin{aligned}
\left\|D^{(1)}(x, \mu)\right\|_{M(\mathbb{C})} & \leq \sum_{n=2}^{\infty}\left\|\int_{0}^{x} e^{(x-2 t) A_{\mu}}\left(\widetilde{T}^{n} \tilde{J}\right)(x, t) d t\right\|_{M(\mathbb{C})} \\
& \leq 3 e^{d} \widetilde{\gamma}(\mu) \sum_{n=2}^{\infty} \frac{e^{2 n d} a_{1}^{n-1}}{(n-2) !}=3 a_{1} e^{5 d} \exp \left(e^{2 d} a_{1}\right) \widetilde{\gamma}(\mu)
\end{aligned}
$$

for all $x \in[0,1]$ and $\mu \in P_{d}$.

The above lemma leads to sharp asymptotic formulas for $D$, which are the main result of this section and were stated in Theorem 1.1.

Proof of Theorem 1.1. Let us start with several simple observations. First of all, remark that clearly

$$
C_{1}\left(\gamma_{q}(\mu)+\gamma(x, \mu)\right) \leq C_{0}
$$

Furthermore, due to inequalities $(2.26),(2.29)$ and (4.11) we get

$$
\begin{aligned}
\left\|R_{0}(x, \mu)\right\|_{M(\mathbb{C})} & \leq\left\|\int_{0}^{x} e^{i \mu(x-2 t)} \tilde{T} \tilde{J}(x, t) d t\right\|_{M(\mathbb{C})}+\left\|D^{(1)}(x, \mu)\right\|_{M(\mathbb{C})} \\
& \leq e^{3 d}\left(a_{2}+1\right)\left(\gamma_{q}(\mu) \gamma(x, \mu)+\gamma_{1}(\mu)\right)+C \widetilde{\gamma}(\mu) \\
& \leq C_{2}\left(\gamma_{q}(\mu) \gamma(x, \mu)+\widetilde{\gamma}(\mu)\right) \\
& \leq 2 C_{2} a_{1} e^{2 d}\left(\gamma(x, \mu)+a_{2} \gamma_{q}(\mu)\right)
\end{aligned}
$$

Note also that from

$$
\left\|\int_{0}^{x} e^{(x-2 t) A_{\mu}} J(t) d t\right\|_{M(\mathbb{C})} \leq e^{d} \gamma(x, \mu), \quad x \in[0,1],
$$

(4.9), and (4.10) it follows that

$$
\left\|D^{(0)}(x, \mu)\right\|_{M(\mathbb{C})} \leq e^{d} \gamma(x, \mu)+2 e^{5 d}\left(1+a_{p}\right) a_{p} \gamma_{q}(\mu), \quad x \in[0,1] .
$$

Combining all these inequalities with Lemma 2.2 and the estimates from (2.26), we prove representations for $D$ from Theorem 1.1. 
Remark 2.3. Note that the explicit formula for $D_{0}$ is the following

$$
\begin{aligned}
& D_{0}(x, \mu)=\left(\begin{array}{ll}
r_{1}(x, \mu) & q_{1}(x, \mu) \\
q_{2}(x, \mu) & r_{2}(x, \mu)
\end{array}\right), \\
& q_{1}(x, \mu):=-\int_{0}^{x} e^{i \mu(x-2 t)} \sigma_{1}(t) d t, \quad q_{2}(x, \mu):=-\int_{0}^{x} e^{-i \mu(x-2 t)} \sigma_{2}(t) d t \\
& r_{1}(x, \mu):=\int_{0}^{x} e^{i \mu(x-2 t)} \tilde{\sigma}_{1}(x, t) d t, \quad r_{2}(x, \mu):=\int_{0}^{x} e^{-i \mu(x-2 t)} \tilde{\sigma}_{2}(x, t) d t
\end{aligned}
$$

and $\tilde{\sigma}_{j}$ are given by (2.17).

Remark 2.4. If $p=1$, then the remainder $R_{0}$ from (1.9) satisfies

$$
\left\|R_{0}(x, \mu)\right\|_{M(\mathbb{C})} \leq C_{2} \Gamma^{2}(\mu),
$$

where $\Gamma$ is given by $(2.24)$.

Remark 2.5. There are misprints in the paper [8] in the formulation of the analogon of Lemma 2.1. The identities (2.14) and (2.15) from [8] should be the same as (2.6) and (2.7) from this paper. Consequently, it implies changes of signs in (2.28), (2.35), (2.37) and inside Remark 2.6 in [8]. Results concerning the spectral problem remains true.

\section{Spectral Problem}

We consider a spectral problem

$$
Y^{\prime}(x)+J(x) Y(x)=A_{\mu} Y(x), \quad x \in[0,1],
$$

associated with the matrix problem (1.1) where $Y=\left[y_{1}, y_{2}\right]^{T}$ and

$$
y_{1}(0)=y_{2}(0), \quad y_{1}(1)=y_{2}(1) .
$$

Let $\mathbf{c}=\mathbf{c}(x, \mu)=\left[c_{1}, c_{2}\right]^{T}$ and $\mathbf{s}=\mathbf{s}(x, \mu)=\left[s_{1}, s_{2}\right]^{T}$ be the solutions of $(3.1)$ satisfying $c_{1}(0)=1, c_{2}(0)=0$ and $s_{1}(0)=0, s_{2}(0)=1$. Then due to conditions (3.2) we find that the eigenvalues are the zeros of

$$
\Phi(\lambda)=c_{1}(1, \lambda)+s_{1}(1, \lambda)-c_{2}(1, \lambda)-s_{2}(1, \lambda) .
$$

The eigenfunctions will be of the form:

$$
Y=\left(\begin{array}{l}
y_{1} \\
y_{2}
\end{array}\right)=\left(\begin{array}{l}
c_{1}\left(\cdot, \mu_{n}\right)+s_{1}\left(\cdot, \mu_{n}\right) \\
c_{2}\left(\cdot, \mu_{n}\right)+s_{2}\left(\cdot, \mu_{n}\right)
\end{array}\right)
$$

The analysis of zeros of (3.3) will lead to the characterization of eigenvalues stated in Theorem 1.2.

Proof. (Proof of Theorem 1.2) The standard approach is to obtain first basic formula for eigenvalues and then derive more accurate form using sharp asymptotic results. We thus need results related to functions $\mathbf{s}$ and $\mathbf{c}$ from (2.6). We derive that

$$
\Phi(\mu)=2 i \sin \mu+\int_{0}^{1} e^{(1-2 t) i \mu}\left(Q_{11}(1, t)+Q_{12}(1, t)-\sigma_{1}(t)\right) d t
$$




$$
-\int_{0}^{1} e^{-(1-2 t) i \mu}\left(Q_{21}(1, t)+Q_{22}(1, t)-\sigma_{2}(t)\right) d t .
$$

Via changing variables in the expression above we obtain

$$
\Phi(\mu)=2 i \sin (\mu)+V(\mu)
$$

where

$$
V(\mu)=\int_{-1}^{1} e^{i \mu s} h(s) d s
$$

and $h$ is a certain function from $L_{p}[-1,1]$.

Note that the identities (3.5) and (3.6) are true not only for $\mu \in P_{d}$ but for all $\mu \in \mathbb{C}$. It is a standard procedure (see for instance [3]) to derive using Rouche Theorem that zeros of $\Phi$ are of the form $\mu_{n}=\pi n+\widetilde{\mu}_{n}$, where $\left(\widetilde{\mu}_{n}\right)$ is bounded. This conclusion implies that eigenvalues lie in a certain horizontal strip of the complex plane. We may continue and investigate more precisely the behavior of $\left(\widetilde{\mu}_{n}\right)$.

The formula for $\Phi$ gives us

$$
\sin \left(\widetilde{\mu}_{n}\right)=\frac{(-1)^{n+1}}{2 i} V\left(\pi n+\widetilde{\mu}_{n}\right)
$$

This expression converges to zero since the convergence of the integral in (3.7) follows from Lebesgue-Riemann Lemma and the fact that $\widetilde{\mu}_{n}$ are bounded. Thus $\widetilde{\mu}_{n} \rightarrow 0$ when $n \rightarrow \infty$. Here ends the reasoning and first claim for $p=1$.

For $1<p<2$ we may continue in order to obtain more information. Using $\sin x=x+\mathrm{O}\left(x^{3}\right), x \rightarrow 0$, and the fact that $\widetilde{\mu}_{n} \rightarrow 0$ we obtain

$$
\widetilde{\mu}_{n}=\frac{(-1)^{n+1}}{2 i} \int_{-1}^{1} e^{i \widetilde{\mu}_{n} s} e^{i \pi n s} h(s) d s+\mathrm{O}\left(\left|\widetilde{\mu}_{n}\right|^{3}\right) .
$$

Next, we recall the expansion of the exponential function

$$
e^{\mu t}=1+\mu t+O\left(|\mu|^{2}\right), \quad \mu \rightarrow 0, \quad|t| \leq 1 .
$$

The above formula and (3.9) yield

$$
\widetilde{\mu}_{n}\left(1-i c_{1, n}-O\left(\left|\widetilde{\mu}_{n}\right|\right)\right)=c_{0, n},
$$

where $c_{j, n}=\int_{0}^{1} e^{i \pi n s} s^{j} h(s) d s$ are Fourier coefficients of $s^{j} h(s), j=0,1$. Since $h \in L_{p}[0,1]$, then by the Hausdorff-Young theorem $\left(c_{j, n}\right) \in l_{q}, j=0,1$. Thus $c_{1, n} \rightarrow 0$ and $\widetilde{\mu}_{n} \rightarrow 0$ as $n \rightarrow \infty$. Formula (3.10) now implies that $\left(\widetilde{\mu}_{n}\right) \in l_{q}$.

Summarizing, we showed that the eigenvalues $\mu_{n}$ of our spectral problem satisfy

$$
\mu_{n}=\pi n+\widetilde{\mu}_{n}, \quad\left(\widetilde{\mu}_{n}\right) \in l_{q}, \quad n \in \mathbb{Z} .
$$

This representation for $1<p$ and the fact that for $p=1$ the remainder goes to zero allows us to find in both cases more accurate description of eigenvalues. Recall we showed that eigenvalues lie in $P_{d}$ for a certain $d>0$, thus we can use asymptotic formulas which are true for $\mu \in P_{d}$. The main tool will be the formulas for $\mathbf{c}$ and $\mathbf{s}$ from Theorem 1.1 and Remark 2.3 and consequently for $\Phi$. 
This way we get

$$
\begin{aligned}
\Phi(\mu) & =2 i \sin \mu-\int_{0}^{1} e^{(1-2 t) i \mu} \sigma_{1}(t) d t+\int_{0}^{1} e^{(1-2 t) i \mu} \tilde{\sigma}_{1}(1, t) d t \\
& +\int_{0}^{1} e^{-(1-2 t) i \mu} \sigma_{2}(t) d t-\int_{0}^{1} e^{-(1-2 t) i \mu} \tilde{\sigma}_{2}(1, t) d t+r(\mu),
\end{aligned}
$$

where

$$
|r(\mu)| \leq c\left(\gamma_{q}(\mu) \gamma(1, \mu)+\widetilde{\gamma}(\mu)\right) \leq c\left(\gamma_{q}^{2}(\mu)+\gamma^{2}(1, \mu)+\widetilde{\gamma}(\mu)\right) .
$$

We now follow along the same lines as in the discussion about eigenvalues to analyze remainders. The representation (3.11) yield

$$
\int_{0}^{1} e^{ \pm(1-2 t) i \mu_{n}} \sigma(t) d t=(-1)^{n} \int_{0}^{1} e^{\mp t i \pi n} e^{ \pm(1-2 t) i \widetilde{\mu}_{n}} \sigma(t) d t
$$

for $\sigma \in L_{p}$. Using an expansion

$$
e^{ \pm(1-2 t) i \widetilde{\mu}_{n}}=1 \pm(1-2 t) i \widetilde{\mu}_{n}+O\left(\left|\widetilde{\mu}_{n}\right|^{2}\right), \quad n \rightarrow \infty,
$$

and Lemma 4.2 we establish

$$
\begin{aligned}
2 i \widetilde{\mu}_{n} & =\int_{0}^{1} e^{-2 \pi i n t} \sigma_{1}(t) d t-\int_{0}^{1} e^{2 \pi i n t} \sigma_{2}(t) d t \\
& +2 \int_{0}^{1} \int_{0}^{t} \sigma_{1}(t) \sigma_{2}(\xi) e^{-2 \pi i n t} e^{2 \pi i n \xi} d \xi d t+r\left(\mu_{n}\right)\left(1+s_{n}+O\left(\left|\widetilde{\mu}_{n}\right|\right)\right),
\end{aligned}
$$

where $s_{n}$ are Fourier coefficients of some functions from $L_{1}[0,1]$. For $p=1$ the last term can be estimated by $\Gamma^{2}(\pi n)$.

Our last aim is to prove that for $\left(r\left(\mu_{n}\right)\right) \in l_{q / 2}$. In what follows we will use a basic formula for eigenvalues (3.11), a simple inequality $\left|e^{i z}-1\right| \leq|z| e^{d}$, $z \in P_{d}$ and the Hausdorff-Young inequality. We infer for $\sigma \in L_{p}[0,1]$ that $1<p<2$

$$
\begin{aligned}
\sum_{n \in \mathbb{Z}}\left|\int_{0}^{x} e^{ \pm 2 i \mu_{n} t} \sigma(t) d t\right|^{q} & \leq c_{q} \sum_{n \in \mathbb{Z}}\left|\int_{0}^{x} e^{ \pm 2 \pi i n t} \sigma(t) d t\right|^{q} \\
& +c_{q} \sum_{n \in \mathbb{Z}}\left(\int_{0}^{x}\left|e^{ \pm 2 i \tilde{\mu}_{n} t}-1 \| \sigma(t)\right| d t\right)^{q} \\
& \leq c_{q}\|\sigma\|_{L_{p}[0,1]}^{q}+c\|\sigma\|_{L_{1}[0,1]}^{q} \sum_{n \in \mathbb{Z}}\left|\tilde{\mu}_{n}\right|^{q} \leq m<\infty,
\end{aligned}
$$

for any $x \in[0,1]$. It follows from (3.16) that

$$
\sup _{x \in[0,1]} \sum_{n \in \mathbb{Z}} \gamma^{q}\left(x, \mu_{n}\right)<\infty,
$$

Note that by (3.17)

$$
\sum_{n \in \mathbb{Z}} \gamma_{q}^{q}\left(\mu_{n}\right) \leq c \int_{0}^{1} \sum_{n \in \mathbb{Z}} \gamma^{q}\left(s, \mu_{n}\right) d s<\infty .
$$


and

$$
\begin{aligned}
\left\|\left(\widetilde{\gamma}\left(\mu_{n}\right)\right)\right\|_{l_{q / 2}} & \leq \int_{0}^{1} \sigma_{0}(s)\left\|\left(\gamma^{2}\left(s, \mu_{n}\right)\right)\right\|_{l_{q / 2}} d s \\
& =\int_{0}^{1} \sigma_{0}(s)\left(\sum_{n \in \mathbb{Z}} \gamma^{q}\left(s, \mu_{n}\right)\right)^{2 / q} d s \leq c\left\|\sigma_{0}\right\|_{L_{1}} .
\end{aligned}
$$

Finally, we obtain

$$
\sum_{n \in \mathbb{Z}}\left|r_{n}\right|^{q / 2}<\infty
$$

Summarizing the discussion above we proved Theorem 1.2.

Remark 3.1. Recall that according to Lemma 4.1 for every $x \in[0,1]$ functions $\tilde{\sigma}_{j}(x, \cdot)$ are from $L_{r}$. If $1<p \leq \frac{4}{3}$, then $1<r \leq 2$ and Fourier coefficients of $\tilde{\sigma}_{j}(x, \cdot)$ are from $l_{q / 2}$. Then the representation (1.10) with

$$
\sum_{n \in \mathbb{Z}}\left|\rho_{n}\right|^{q / 2}<\infty
$$

is true but with $\mu_{0, n}$ given by

$$
2 i \mu_{0, n}=\int_{0}^{1} e^{-2 \pi i n t} \sigma_{1}(t) d t-\int_{0}^{1} e^{2 \pi i n t} \sigma_{2}(t) d t .
$$

Now, we can proceed to eigenfunctions. We are going to combine results from Theorem 1.2 with Lemma 2.2 and Theorem 1.1 .

Theorem 3.2. Let $1<p<2$ and

$$
\begin{aligned}
& F_{1}(x, t)=-\sigma_{1}(t)+\tilde{\sigma}_{1}(x, t)-\left(T_{\sigma_{1}} \tilde{\sigma}_{2}\right)(x, t) \\
& F_{2}(x, t)=-\sigma_{2}(t)+\tilde{\sigma}_{2}(x, t)-\left(T_{\sigma_{2}} \tilde{\sigma}_{1}\right)(x, t) .
\end{aligned}
$$

The eigenfunctions of the spectral problem (3.1)-(3.2) admit the representation

$$
\begin{aligned}
y_{1}\left(x, \mu_{n}\right) & =e^{i \pi n x}\left(1+i \mu_{0, n} x\right)\left(1+\int_{0}^{x} e^{-2 \pi i n t} F_{1}(x, t) d t\right) \\
& -2 i \mu_{0, n} e^{i \pi n x} \int_{0}^{x} e^{-2 \pi i n t} t F_{1}(x, y) d t+r_{1}(x, n), \\
y_{2}\left(x, \mu_{n}\right) & =e^{-i \pi n x}\left(1-i \mu_{0, n} x\right)\left(1+\int_{0}^{x} e^{2 \pi i n t} F_{2}(x, t) d t\right) \\
& +2 i \mu_{0, n} e^{-i \pi n x} \int_{0}^{x} e^{2 \pi i n t} t F_{2}(x, t) d t+r_{2}(x, n),
\end{aligned}
$$

where

$$
\sum_{n \in \mathbb{Z}} \sup _{x \in[0,1]}\left|r_{j}(x, n)\right|^{q / 2}<\infty
$$


Proof. According to (3.4) eigenfunctions are expressed by solutions $\mathbf{c}$ and $\mathbf{s}$ in the following way

$$
y_{1}\left(x, \mu_{n}\right)=c_{1}\left(x, \mu_{n}\right)+s_{1}\left(x, \mu_{n}\right)
$$

and

$$
y_{2}\left(x, \mu_{n}\right)=c_{2}\left(x, \mu_{n}\right)+s_{2}\left(x, \mu_{n}\right) .
$$

The formula (2.28) yields that

$$
\begin{aligned}
y_{1}\left(x, \mu_{n}\right) & =e^{i \mu_{n} x}-\int_{0}^{x} e^{(x-2 t) i \mu_{n}} \sigma_{1}(t) d t \\
& +\int_{0}^{x} e^{(x-2 t) i \mu_{n}} \tilde{\sigma}_{1}(x, t) d t-\int_{0}^{x} e^{i \mu(x-2 t)}\left(T_{\sigma_{1}} \tilde{\sigma}_{2}\right)(x, t) d t+\alpha\left(x, \mu_{n}\right), \\
y_{2}\left(x, \mu_{n}\right) & =e^{-i \mu_{n} x}-\int_{0}^{x} e^{-(x-2 t) i \mu_{n}} \sigma_{2}(t) d t+\int_{0}^{x} e^{-(x-2 t) i \mu_{n}} \tilde{\sigma}_{2}(x, t) d t \\
& -\int_{0}^{x} e^{-i \mu(x-2 t)}\left(T_{\sigma_{2}} \tilde{\sigma}_{1}\right)(x, t) d t+\beta\left(x, \mu_{n}\right),
\end{aligned}
$$

where

$$
\left|\alpha\left(x, \mu_{n}\right)\right|+\left|\beta\left(x, \mu_{n}\right)\right| \leq c \widetilde{\gamma}\left(\mu_{n}\right) .
$$

Repeating once more all arguments used in order to derive formulas for eigenvalues, we obtain the thesis with claimed estimates for remainders.

It is possible to obtain shorter but less precise formulas for eigenfunctions. This time we use the representation (1.9) and comments from Lemma 4.2 to prove the following fact.

Corollary 3.3. Let $1 \leq p<2$, then the eigenfunctions of the spectral problem (3.1)-(3.2) admit the representation

$$
\begin{aligned}
y_{1}\left(x, \mu_{n}\right) & =e^{i \pi n x}\left(1+i \mu_{0, n} x-\int_{0}^{x} e^{-2 \pi i n t} \sigma_{1}(t) d t\right. \\
& \left.+\int_{0}^{x} \int_{0}^{s} \sigma_{1}(s) \sigma_{2}(\xi) e^{-2 i \mu s} e^{2 i \mu \xi} d \xi d s\right)+r_{1}(x, n), \\
y_{2}\left(x, \mu_{n}\right) & =e^{-i \pi n x}\left(1-i \mu_{0, n} x-\int_{0}^{x} e^{2 \pi i n t} \sigma_{2}(t) d t\right. \\
& \left.+\int_{0}^{x} \int_{0}^{s} \sigma_{1}(\xi) \sigma_{2}(s) e^{2 i \mu s} e^{-2 i \mu \xi} d \xi d s\right)+r_{2}(x, n),
\end{aligned}
$$

where for $1<p<2$ we have

$$
\sup _{x \in[0,1]} \sum_{n \in \mathbb{Z}}\left|r_{j}(x, n)\right|^{q / 2}<\infty,
$$

whereas for $p=1$ there holds

$$
\left|r_{j}(x, n)\right| \leq c \Gamma^{2}(\pi n) .
$$




\section{Acknowledgements}

The author is grateful to the referee for careful reading of the paper and very helpful comments and remarks, which improved the paper significantly. This work was supported by NCN grant no. UMO-2017/27/B/ST1/00078.

Open Access. This article is licensed under a Creative Commons Attribution 4.0 International License, which permits use, sharing, adaptation, distribution and reproduction in any medium or format, as long as you give appropriate credit to the original author(s) and the source, provide a link to the Creative Commons licence, and indicate if changes were made. The images or other third party material in this article are included in the article's Creative Commons licence, unless indicated otherwise in a credit line to the material. If material is not included in the article's Creative Commons licence and your intended use is not permitted by statutory regulation or exceeds the permitted use, you will need to obtain permission directly from the copyright holder. To view a copy of this licence, visit http:// creativecommons.org/licenses/by/4.0/.

Publisher's Note Springer Nature remains neutral with regard to jurisdictional claims in published maps and institutional affiliations.

\section{Appendix}

Lemma 4.1. For every $x \in[0,1]$ and $j=1,2$ the functions $\tilde{\sigma}_{j}(x, \cdot)$ belong to $L_{r}[0,1]$, where $r$ is defined in (2.1). Furthermore, we have $\tilde{\sigma}_{j} \in B, j=1,2$, and $\tilde{J} \in M(B)$.

Proof. We take $(x, t) \in \Delta$. Let $\widehat{\sigma}_{1}, \widehat{\sigma}_{2}$ denote the extension of $\sigma_{1}$ and $\sigma_{2}$ by zero outside $[0,1]$. Note that for every $x \in[0,1]$ we get

$$
\begin{aligned}
\int_{0}^{x-t} \sigma_{1}(t+\xi) \sigma_{2}(\xi) d \xi & =\int_{-\infty}^{\infty} \widehat{\sigma}_{1}(t+\xi) \widehat{\sigma}_{2}(\xi) \chi(x-(t+\xi)) d \xi \\
& =\int_{-\infty}^{\infty} \widehat{\sigma}_{1}(t-s) \widehat{\sigma}_{2}(-s) \chi(x-(t-s)) d s \\
& =\left(\left(\widehat{\sigma}_{1}(\cdot) \chi(x-\cdot)\right) *\left(\widehat{\sigma}_{2}(-\cdot)\right)\right)(t) .
\end{aligned}
$$

We thus have

$$
\begin{aligned}
\left\|\int_{0}^{x-t} \sigma_{1}(t+\xi) \sigma_{2}(\xi) d \xi\right\|_{L_{r}[0,1]} & \leq\left\|\int_{0}^{x-t} \widehat{\sigma}_{1}(t+\xi) \widehat{\sigma}_{2}(\xi) d \xi\right\|_{L_{r}(\mathbb{R})} \\
& \leq\left\|\left(\widehat{\sigma}_{1}(\cdot) \chi(x-\cdot)\right)\right\|_{L_{p}(\mathbb{R})}\left\|\widehat{\sigma}_{2}(-\cdot)\right\|_{L_{p}(\mathbb{R})} \\
& \leq\left\|\sigma_{1}\right\|_{L_{p}[0,1]}\left\|\sigma_{2}\right\|_{L_{p}[0,1]}
\end{aligned}
$$

hence

$$
\left\|\tilde{\sigma}_{j}\right\|_{B} \leq\left\|\sigma_{1}\right\|_{L_{p}[0,1]}\left\|\sigma_{2}\right\|_{L_{p}[0,1]}, \quad j=1,2 .
$$

Clearly, a similar estimate holds for $\tilde{\sigma}_{2}$ as well. 
Therefore, if we consider $\epsilon$ and $x$ such that $0 \leq t \leq x+\epsilon \leq 1$, then using again the inequality for convolutions, we obtain

$$
\begin{aligned}
& \left\|\int_{0}^{x+\epsilon-t} \sigma_{1}(t+\xi) \sigma_{2}(\xi) d \xi-\int_{0}^{x-t} \sigma_{1}(t+\xi) \sigma_{2}(\xi) d \xi\right\|_{L_{r}[0,1]} \\
& \quad \leq \|\left(\widehat{\sigma}_{1}(\cdot)[\chi(x+\epsilon-\cdot)-\chi(x-\cdot)]\left\|_{L_{p}(\mathbb{R})}\right\| \widehat{\sigma}_{2}(-\cdot) \|_{L_{p}(\mathbb{R})}\right. \\
& \quad \leq \int_{\mathbb{R}}\left|\widehat{\sigma}_{1}(s)\right|^{p}|\chi(x+\epsilon-s)-\chi(x-s)|^{p} d s\left\|\widehat{\sigma}_{2}\right\|_{L_{p}(\mathbb{R})} .
\end{aligned}
$$

The integral in the last line converges to zero, if $\epsilon \rightarrow 0$, because of Lebesgue Theorem, hence the mapping $x \mapsto \tilde{\sigma}_{j}(x, \cdot) \in L_{r}[0,1]$ is continuous for $j=1,2$.

Lemma 4.2. The following identity holds

$\int_{0}^{x} e^{-2 t i \mu} \tilde{\sigma}_{1}(x, t) d t+\int_{0}^{x} e^{2 t i \mu} \tilde{\sigma}_{2}(x, t) d t=\int_{0}^{x} e^{-2 i \mu \xi} \sigma_{1}(\xi) d \xi \int_{0}^{x} \sigma_{2}(s) e^{2 i \mu s} d s$.

Moreover, we have

$$
\left|\int_{0}^{x} e^{-2 t i \mu} \tilde{\sigma}_{1}(x, t) d t+\int_{0}^{x} e^{2 t i \mu} \tilde{\sigma}_{2}(x, t) d t\right| \leq c \gamma^{2}(x, \mu)
$$

and

$$
\begin{aligned}
& \int_{0}^{x} e^{-2 t i \mu} \tilde{\sigma}_{1}(x, t) d t-\int_{0}^{x} e^{2 t i \mu} \tilde{\sigma}_{2}(x, t) d t \\
& \quad=-2 \int_{0}^{x} \int_{0}^{s} \sigma_{1}(\xi) \sigma_{2}(s) e^{2 i \mu s} e^{-2 i \mu \xi} d \xi d s+\alpha(\mu),
\end{aligned}
$$

where $\alpha(\mu)=O\left(\gamma^{2}(x, \mu)\right)$.

Proof. Note that

$$
\begin{aligned}
\int_{0}^{x} e^{-2 t i \mu} \tilde{\sigma}_{1}(x, t) d t & =\int_{0}^{x} \int_{0}^{s} \sigma_{1}(s) \sigma_{2}(\xi) e^{-2 i \mu s} e^{2 i \mu \xi} d \xi d s \\
\int_{0}^{x} e^{2 t i \mu} \tilde{\sigma}_{2}(x, t) d t & =\int_{0}^{x} \int_{0}^{s} \sigma_{1}(\xi) \sigma_{2}(s) e^{2 i \mu s} e^{-2 i \mu \xi} d \xi d s .
\end{aligned}
$$

Observe that the change of variables yields

$$
\begin{aligned}
\int_{0}^{x} e^{-2 t i \mu} \tilde{\sigma}_{1}(x, t) d t & =\int_{0}^{x} \int_{\xi}^{x} \sigma_{1}(\xi) \sigma_{2}(s) e^{-2 i \mu s} e^{2 i \mu \xi} d s d \xi \\
& =\int_{0}^{x} \int_{s}^{x} \sigma_{1}(s) \sigma_{2}(\xi) e^{-2 i \mu \xi} e^{2 i \mu s} d \xi d s
\end{aligned}
$$

thus

$$
\begin{aligned}
\int_{0}^{x} e^{-2 t i \mu} \tilde{\sigma}_{1}(x, t) d t & +\int_{0}^{x} e^{2 t i \mu} \tilde{\sigma}_{2}(x, t) d t \\
& =\int_{0}^{x} \int_{0}^{x} \sigma_{1}(\xi) \sigma_{2}(s) e^{2 i \mu s} e^{-2 i \mu \xi} d \xi d s \\
& =\int_{0}^{x} e^{-2 i \mu \xi} \sigma_{1}(\xi) d \xi \int_{0}^{x} \sigma_{2}(s) e^{2 i \mu s} d s
\end{aligned}
$$


This equality implies that

$$
\left|\int_{0}^{x} e^{-2 t i \mu} \tilde{\sigma}_{1}(x, t) d t+\int_{0}^{x} e^{2 t i \mu} \tilde{\sigma}_{2}(x, t) d t\right| \leq c \gamma^{2}(x, \mu) .
$$

What is more, then

$$
\begin{aligned}
\int_{0}^{x} e^{-2 t i \mu} \tilde{\sigma}_{1}(x, t) d t & -\int_{0}^{x} e^{2 t i \mu} \tilde{\sigma}_{2}(x, t) d t \\
& =\int_{0}^{x} e^{-2 i \mu \xi} \sigma_{1}(\xi) d \xi \int_{0}^{x} \sigma_{2}(s) e^{2 i \mu s} d s \\
& -2 \int_{0}^{x} \int_{0}^{s} \sigma_{1}(\xi) \sigma_{2}(s) e^{2 i \mu s} e^{-2 i \mu \xi} d \xi d s
\end{aligned}
$$

thus

$$
\begin{aligned}
\int_{0}^{x} e^{-2 t i \mu} \tilde{\sigma}_{1}(x, t) d t & -\int_{0}^{x} e^{2 t i \mu} \tilde{\sigma}_{2}(x, t) d t \\
& =-2 \int_{0}^{x} \int_{0}^{s} \sigma_{1}(\xi) \sigma_{2}(s) e^{2 i \mu s} e^{-2 i \mu \xi} d \xi d s \\
& +\alpha(\mu)
\end{aligned}
$$

where $\alpha(\mu)=O\left(\gamma^{2}(x, \mu)\right)$.

Lemma 4.3. The linear operator $T_{\sigma}$

$$
\left(T_{\sigma} f\right)(x, t)=\int_{0}^{x-t} \sigma(t+\xi) f(t+\xi, \xi) d \xi=\int_{t}^{x} \sigma(s) f(s, s-t) d s
$$

where $\sigma \in L_{p}[0,1]$, is bounded in $B$.

Proof. Note that

$$
\begin{aligned}
\left(\int_{0}^{x}\left|\left(T_{\sigma} f\right)(x, t)\right|^{r} d t\right)^{1 / r} & =\left(\int_{0}^{x}\left|\int_{0}^{x} \chi(s-t) \sigma(s) f(s, s-t) d s\right|^{r} d t\right)^{1 / r} \\
& \leq \int_{0}^{x}|\sigma(s)|\left(\int_{0}^{s}|f(s, s-t)|^{r} d t\right)^{1 / r} d s \\
& \leq \int_{0}^{x}|\sigma(s)|\left(\int_{0}^{s}|f(s, \tau)|^{r} d \tau\right)^{1 / r} d s \\
& \leq \int_{0}^{x}|\sigma(s)| d s \sup _{s \in[0,1]}\left(\int_{0}^{s}|f(s, \tau)|^{r} d \tau\right)^{1 / r} \\
& \leq\|\sigma\|_{L_{1}}\|f\|_{B} .
\end{aligned}
$$

For the proof of continuity we take $\epsilon$ and $x$ such that $0 \leq t \leq x+\epsilon \leq 1$. Then

$$
\begin{aligned}
\left\|\left(T_{\sigma} f\right)(x+\epsilon, \cdot)-\left(T_{\sigma} f\right)(x, \cdot)\right\|_{L_{r}[0,1]} & \leq\left(\int_{0}^{x}\left|\int_{x}^{x+\epsilon} \sigma(s) f(s, s-t) d s\right|^{r} d t\right)^{1 / r} \\
& +\left(\int_{x}^{x+\epsilon}\left|\int_{t}^{x+\epsilon} \sigma(s) f(s, s-t) d s\right|^{r} d t\right)^{1 / r} .
\end{aligned}
$$


First integral can be estimated as follows

$$
\begin{aligned}
\left(\int_{0}^{x}\left|\int_{x}^{x+\epsilon} \sigma(s) f(s, s-t) d s\right|^{r} d t\right)^{1 / r} & \leq \int_{x}^{x+\epsilon}|\sigma(s)|\left(\int_{0}^{x}|f(s, s-t)|^{r} d t\right)^{1 / r} d s \\
& \leq \int_{x}^{x+\epsilon}|\sigma(s)|\left(\int_{0}^{s}|f(s, \tau)|^{r} d \tau\right)^{1 / r} d s \\
& \leq\|f\|_{B} \int_{x}^{x+\epsilon}|\sigma(s)| d s
\end{aligned}
$$

and this expression goes to zero whenever $\epsilon$ does.

Second integral can be treated in an analogous way, hence the proof is completed.

Lemma 4.4. The operators $T_{k j}, k, j=1,2, k \neq j$ satisfy the following estimate

$$
\left\|T_{k j}^{n} f\right\|_{B} \leq \frac{a^{n}}{n !}\|f\|_{B}, \quad f \in B, \quad n \in \mathbb{N}, \quad k, j=1,2, \quad k \neq j .
$$

Proof. Consider the operator $T_{12}$. Note that directly from the third line of (4.3) we get

$$
\begin{aligned}
\left(\int_{0}^{x}\left|\left(T_{12} f\right)(x, t)\right|^{r} d t\right)^{1 / r} & \leq \int_{0}^{x}\left|\sigma_{1}(s)\right|\left(\int_{0}^{s}\left|\left(T_{\sigma_{2}} f\right)(s, \tau)\right|^{r} d \tau\right)^{1 / r} d s \\
& \leq \int_{0}^{x}\left|\sigma_{1}(s)\right| \int_{0}^{s}\left|\sigma_{2}(\tau)\right|\left(\int_{0}^{\tau}|f(\tau, \xi)|^{r} d \xi\right)^{1 / r} d \tau d s \\
& \leq\|f\|_{B} \int_{0}^{x}|\sigma(s)| \int_{0}^{s}\left|\sigma_{2}(\tau)\right| d s .
\end{aligned}
$$

Define $\eta \in C[0,1]$ by

$$
\eta(x):=\int_{0}^{x}\left|\sigma_{1}(s)\right|\left(\int_{0}^{s}\left|\sigma_{2}(\tau)\right| d \tau\right) d s, \quad x \in[0,1] .
$$

This function is increasing and bounded by $a=\left\|\sigma_{1}\right\|_{L_{1}}\left\|\sigma_{2}\right\|_{L_{1}}$. It suffices to prove that for all $(x, t) \in \Delta$ and $n=1,2, \ldots$,

$$
\left(\int_{0}^{x}\left|\left(T_{12}^{n} f\right)(x, t)\right|^{r} d t\right)^{1 / r} \leq \frac{\|f\|_{B}}{n !} \eta^{n}(x), \quad f \in B .
$$

For $n=1$ the estimate (4.6) was shown above. Arguing by induction, suppose that (4.6) holds for some $n \in \mathbb{N}$. Then, for $(x, t) \in \Delta$ and $f \in B$, by (4.5) we have

$$
\begin{aligned}
& \left(\int_{0}^{x}\left|\left(T_{12}^{n+1} f\right)(x, t)\right|^{r} d t\right)^{1 / r} \\
& \quad \leq \int_{0}^{x}\left|\sigma_{1}(s)\right| \int_{0}^{s}\left|\sigma_{2}(\tau)\right|\left(\int_{0}^{\tau}\left|\left(T_{12}^{n} f\right)(\tau, \xi)\right|^{r} d \xi\right)^{1 / r} d \tau d s \\
& \quad \leq \frac{\|f\|_{B}}{n !} \int_{0}^{x}\left|\sigma_{1}(s)\right| \int_{0}^{s}\left|\sigma_{2}(\tau)\right| \eta^{n}(\tau) d \tau d s \\
& \quad \leq \frac{\|f\|_{B}}{n !} \int_{0}^{x}\left|\sigma_{1}(s)\right| \int_{0}^{s}\left|\sigma_{2}(\tau)\right| d \tau \eta^{n}(s) d s
\end{aligned}
$$




$$
=\frac{\|f\|_{B}}{n !} \int_{0}^{x} \eta^{n}(s) d \eta(s)=\frac{\|f\|_{B}}{(n+1) !} \eta^{n+1}(x) .
$$

Therefore (4.6) is true and then after taking supremum over $x \in[0,1]$ we get (4.4).

Next proposition we state below without a proof, since it can be found in [8, Prop. 6.1].

Proposition 4.5. If $\sigma_{j} \in L_{p}[0,1], 1 \leq p<2$ and $F \in M(B)$, then

$$
\int_{0}^{x} e^{-2 i \mu t}(\widetilde{T} F)(x, t) d t=-\int_{0}^{x} e^{-2 i \mu s} J(s) \int_{0}^{s} e^{2 i \mu \xi} F(s, \xi) d \xi d s .
$$

Moreover,

$$
\begin{aligned}
\int_{0}^{x} e^{-2 i \mu t} & (\widetilde{T} \tilde{J})(x, t) d t \\
& =-\int_{0}^{x} e^{2 i \mu y}\left(\int_{y}^{x} J(z) e^{-2 i \mu z} d z \int_{0}^{y} J^{T}(\tau) e^{-2 i \mu \tau} d \tau\right) J^{T}(y) d y .
\end{aligned}
$$

Lemma 4.6. If $\mu \in P_{d}$, and $x \in[0,1]$, then the following inequalities hold

$$
\begin{aligned}
& \left\|\int_{0}^{x} e^{-2 t A_{\mu}} \tilde{J}(x, t) d t\right\|_{M(\mathbb{C})} \leq 2 e^{2 d} a_{p} \gamma_{q}(\mu) \\
& \left\|\int_{0}^{x} e^{-2 t A_{\mu}}(\widetilde{T} \tilde{J})(x, t) d t\right\|_{M(\mathbb{C})} \leq 2 e^{4 d} a_{p}^{2} \gamma_{q}(\mu) \\
& \left\|\int_{0}^{x} e^{-2 t A_{\mu}}(\widetilde{T} \tilde{J})(x, t) d t\right\|_{M(\mathbb{C})} \leq 2\left(a_{p}+1\right) e^{2 d}\left(\gamma_{q}(\mu) \gamma(x, \mu)+\widetilde{\gamma}(\mu)\right), \\
& \left\|\int_{0}^{x} e^{-2 t A_{\mu}}\left(\widetilde{T}^{n} \tilde{J}\right)(x, t) d t\right\|_{M(\mathbb{C})} \leq 3 e^{2 n d} \frac{a_{1}^{n-1}}{(n-2) !} \widetilde{\gamma}(\mu), \quad n \geq 2
\end{aligned}
$$

Proof. Note that

$$
\begin{aligned}
\left\|\int_{0}^{x} e^{-2 i \mu t} \tilde{J}(x, t) d t\right\|_{M(\mathbb{C})} & =\left\|\int_{0}^{x} J(s) e^{-2 i \mu s} \int_{0}^{s} J(\xi) e^{2 i \mu \xi} d \xi d s\right\|_{M(\mathbb{C})} \\
& =\left|\int_{0}^{x} e^{-2 i \mu s} \sigma_{1}(s) \int_{0}^{s} e^{2 i \mu \xi} \sigma_{2}(\xi) d \xi d s\right| \\
& +\left|\int_{0}^{x} e^{-2 i \mu s} \sigma_{2}(s) \int_{0}^{s} e^{2 i \mu \xi} \sigma_{1}(\xi) d \xi d s\right| \\
& \leq e^{2 d}\left\{\left\|\sigma_{1}\right\|_{L_{p}}\left\|\int_{0}^{s} e^{2 i \mu \xi} \sigma_{2}(\xi) d \xi\right\|_{L_{q}}\right. \\
& \left.+\left\|\sigma_{2}\right\|_{L_{p}}\left\|\int_{0}^{s} e^{2 i \mu \xi} \sigma_{1}(\xi) d \xi\right\|_{L_{q}}\right\} \\
& \leq e^{2 d} \max \left\{\left\|\sigma_{1}\right\|_{L_{p}},\left\|\sigma_{2}\right\|_{L_{p}}\right\} \gamma_{q}(\mu), \quad x \in[0,1] .
\end{aligned}
$$


We thus proved the estimate (4.9).

Next, by (4.7), if $\mu \in P_{d}, x \in[0,1]$ and $F \in M(B)$, then

$$
\left\|\int_{0}^{x} e^{-2 i \mu t}(\widetilde{T} F)(x, t) d t\right\|_{M(\mathbb{C})} \leq e^{2 d} \int_{0}^{x}\left\|J(s) \int_{0}^{s} e^{2 i \mu \xi} F(s, \xi) d \xi\right\|_{M(\mathbb{C})} d s
$$

and

$$
\left\|\int_{0}^{x} e^{-2 i \mu t}(\widetilde{T} F)(x, t) d t\right\|_{M(\mathbb{C})} \leq e^{2 d} a_{1}\left\|\int_{0}^{s} e^{2 i \mu \xi} F(s, \xi) d \xi\right\|_{M(\mathbb{C})} .
$$

We use (4.15) and (4.13) to obtain that

$$
\begin{aligned}
\left\|\int_{0}^{x} e^{-2 i \mu t}(\widetilde{T} \tilde{J})(x, t) d t\right\|_{M(\mathbb{C})} & \leq e^{2 d} a_{1}\left\|\int_{0}^{s} e^{2 i \mu \xi} \tilde{J}(s, \xi) d \xi\right\|_{M(\mathbb{C})} \\
& \leq e^{4 d} a_{p}^{2} \gamma_{q}(\mu),
\end{aligned}
$$

thus, the estimate (4.10) holds.

Due to the estimate

$$
\left|\int_{0}^{x} \sigma_{0}(s) \gamma(y, \mu) d y\right| \leq\left\|\sigma_{0}\right\|_{L_{p}}\|\gamma(y, \mu)\|_{L_{q}} \leq a_{2} \gamma_{q}(\mu)
$$

the inequality (4.11) holds if

$$
\left\|\int_{0}^{x} e^{-2 i \mu t}(\widetilde{T} \tilde{J})(x, t) d t\right\|_{M(\mathbb{C})} \leq e^{2 d}\left(\widetilde{\gamma}(\mu)+\gamma(x, \mu) \int_{0}^{x} \sigma_{0}(y) \gamma(y, \mu) d y\right) .
$$

Whereas, using (4.8), (4.9), we have

$$
\begin{aligned}
& \left\|\int_{0}^{x} e^{-2 i \mu t}(\widetilde{T} \tilde{J})(x, t) d t\right\|_{M(\mathbb{C})} \\
& \leq e^{2 d} \int_{0}^{x}\left\|\int_{y}^{x} e^{-2 i \mu z} J(z) d z \int_{0}^{y} e^{-2 i \mu \tau} J^{T}(\tau) d \tau J^{T}(y)\right\|_{M(\mathbb{C})} d y \\
& \leq e^{2 d} \int_{0}^{x} \sigma_{0}(y)\left\|\int_{y}^{x} e^{-2 i \mu z} J(z) d z\right\|_{M(\mathbb{C})}\left\|\int_{0}^{y} e^{-2 i \mu \tau} J(\tau) d \tau\right\|_{M(\mathbb{C})} d y \\
& \leq e^{2 d} \int_{0}^{x} \sigma_{0}(y)\left\|\int_{0}^{y} e^{-2 i \mu \tau} J(\tau) d \tau\right\|_{M(\mathbb{C})}^{2} d y \\
& \quad+e^{2 d}\left\|\int_{0}^{x} e^{-2 i \mu z} J(z) d z\right\| \int_{M(\mathbb{C})}^{x} \sigma_{0}(y)\left\|\int_{0}^{y} e^{-2 i \mu \tau} J(\tau) d \tau\right\|_{M(\mathbb{C})} d y \\
& \leq e^{2 d} \widetilde{\gamma}(\mu)+e^{2 d} \gamma(x, \mu) \int_{0}^{x} \sigma_{0}(y) \gamma(y, \mu) d y,
\end{aligned}
$$

and (4.16) follows. 
The estimate (4.12) will be showed, if we prove that for all $n \geq 2$ and any $x \in[0,1]$,

$$
\left\|\int_{0}^{x} e^{-2 i \mu t}\left(\widetilde{T}^{n} \tilde{J}\right)(x, t) d t\right\|_{M(\mathbb{C})} \leq \frac{3}{2} \frac{e^{2 n d}}{(n-2) !}\left(\int_{0}^{x} \sigma_{0}(s) d s\right)^{n-2} a_{1} \widetilde{\gamma}(\mu) .
$$

We proceed by induction. Using (4.14) for $F=\tilde{T} \tilde{J}$ and (4.16), we note that

$$
\begin{aligned}
& \left\|\int_{0}^{x} e^{-2 i \mu t}\left(\widetilde{T}^{2} \tilde{J}\right)(x, t) d t\right\|_{M(\mathbb{C})} \\
& \quad \leq e^{2 d} \int_{0}^{x} \sigma_{0}(s)\left\|\int_{0}^{s} e^{2 i \mu \xi}(\widetilde{T} \tilde{J})(s, \xi) d \xi\right\|_{M(\mathbb{C})} d s \\
& \quad \leq e^{4 d} \int_{0}^{x} \sigma_{0}(s)\left(\gamma(s, \mu) \int_{0}^{s} \sigma_{0}(y) \gamma(y, \mu) d y+\widetilde{\gamma}(\mu)\right) d s \\
& \quad \leq e^{4 d} \int_{0}^{x} \sigma_{0}(s) \gamma(s, \mu) \int_{0}^{s} \sigma_{0}(y) \gamma(y, \mu) d y d s+e^{4 d} a_{1} \widetilde{\gamma}(\mu) \\
& \quad \leq e^{4 d} \frac{\left(\int_{0}^{x} \sigma_{0}(s) \gamma(s, \mu) d s\right)^{2}}{2}+e^{4 d} a_{1} \widetilde{\gamma}(\mu) .
\end{aligned}
$$

Since $\sigma_{0}$ is nonnegative we may now apply Cauchy-Bunyakovsky-Schwarz inequality to obtain

$$
\left(\int_{0}^{x} \sigma_{0}(s) \gamma(s, \mu) d s\right)^{2} \leq \int_{0}^{x} \sigma_{0}(s) d s \int_{0}^{x} \sigma_{0}(s) \gamma^{2}(s, \mu) d s \leq a_{1} \widetilde{\gamma}(\mu) .
$$

Therefore, we proved

$$
\left\|\int_{0}^{x} e^{-2 i \mu t}\left(\widetilde{T}^{2} \tilde{J}\right)(x, t) d t\right\|_{M(\mathbb{C})} \leq \frac{3}{2} e^{4 d} a_{1} \widetilde{\gamma}(\mu)
$$

hence (4.17) holds for $n=2$.

Suppose that (4.17) holds for some $n \geq 2$. We use (4.14) to derive

$$
\begin{aligned}
& \left\|\int_{0}^{x} e^{-2 i \mu t}\left(\widetilde{T}^{n+1} \tilde{J}\right)(x, t) d t\right\|_{M(\mathbb{C})} \\
& \quad \leq e^{2 d} \int_{0}^{x} \sigma_{0}(s)\left\|\int_{0}^{s} e^{2 i \mu \xi}\left(\widetilde{T}^{n} \tilde{J}\right)(s, \xi) d \xi\right\|_{M(\mathbb{C})} d s \\
& \quad \leq \frac{3}{2} \frac{e^{2(n+1) d}}{(n-2) !} a_{1} \widetilde{\gamma}(\mu) \int_{0}^{x} \sigma_{0}(s)\left(\int_{0}^{s} \sigma_{0}(\tau) d \tau\right)^{n-2} d s \\
& \quad=\frac{3}{2} \frac{e^{2(n+1) d}}{(n-1) !} a_{1} \widetilde{\gamma}(\mu)\left(\int_{0}^{x} \sigma_{0}(\tau) d \tau\right)^{n-1}, \quad x \in[0,1],
\end{aligned}
$$

thus (4.17) holds also for $n+1$, and the proof of (4.17) is completed. 


\section{References}

[1] Agibalova, A.V., Malamud, M.M., Oridoroga, L.L.: On the completeness of general boundary value problems for $2 \times 2$ first-order systems of ordinary differential equations. Methods Funct. Anal. Topol. 18, 4-18 (2012)

[2] Albeverio, S., Hryniv, R., Mykytyuk, Y.: Inverse spectral problems for Dirac operators with summable potentials. Rus. J. Math. Phys. 12, 406-423 (2005)

[3] Bellman, R., Cook, K.L.: Differ. Differ. Equ. Academic Press, New York (1963)

[4] Birkhoff, G.D., Langer, R.E.: The boundary problems and developments associated with a system of ordinary differential equations of the first order. Proc. Amer. Acad. Arts Sci. 58, 49-128 (1923)

[5] Djakov, P., Mityagin, B.: Bari-Markus property for Riesz projections of 1D periodic Dirac operators. Mat. Nachr. 283, 443-462 (2010)

[6] Djakov, P., Mityagin, B.: Unconditional convergence of spectral decompositions of 1D Dirac operators with regular boundary conditions. Indiana Univ. Math. J. 61, 359-398 (2012)

[7] Djakov, P., Mityagin, B.: Riesz bases consisting of root functions of 1D Dirac operators. Proc. Amer. Math. Soc. 141, 1361-1375 (2013)

[8] Gomilko, A.M., Rzepnicki, E: On asymptotic behaviour of solutions of the Dirac system and applications to the Sturm-Liouville problem with a singular potential. J. Spectr. Theory 10, 747-786 (2020)

[9] Levitan, B.M., Sargsyan, I.S.: Sturm-Liouville and Dirac Operators. Kluwer Academic Publishers Group, Dordrecht (1991)

[10] Lunyov, A.A., Malamud. M.M.: Stability of spectral characteristics and Bari basis property of boundary value problems for $2 \times 2$ Dirac type systems. arXiv:2012.11170

[11] Lunyov, A.A., Malamud, M.M.: On the Riesz basis property of the root vector system for Dirac-Type $2 \times 2$ Systems. Dokl. Math. 90, 556-561 (2014)

[12] Lunyov, A.A., Malamud, M.M.: On the completeness of and Riesz basis property of root subspaces of boundary value problems for first order systems and applications. JST 5, 17-70 (2015)

[13] Lunyov, A.A., Malamud, M.M.: On the Riesz basis property of root vectors system for $2 \times 2$ Dirac type operators. J. Math. Anal. Appl. 441, 57-103 (2016)

[14] Malamud, M.M., Oridoroga, L.L.: Completeness theorems for systems of differential equations. Funct. Anal. Appl. 34, 308-310 (2000)

[15] Malamud, M.M., Oridoroga, L.L.: On the completeness of root subspaces of boundary value problems for first order systems of ordinary differential equations. J. Funct. Anal. 263, 1939-1980 (2012)

[16] Marchenko, V.A.: Sturm-Liouville Operators and Their Applications. Birkhauser, Basel (1986)

[17] Puyda, D.V.: Inverse spectral problems for dirac operators with summable matrix-valued potentials. Integr. Equ. Oper. Theory 74, 417-450 (2012)

[18] Rykhlov, V.S.: Asymptotical formulas for solutions of linear differential systems of the first order. Results Math. 36, 342-353 (1999)

[19] Sadovnichaya, I.V.: Uniform asymptotics of the eigenvalues and eigenfunctions of the Dirac system with an integrable potential. Differ. Equ. 52, 1000-1010 (2016) 
[20] Savchuk, A.M., Sadovnichaya, I.V.: Asymptotic formulas for fundamental solutions of the dirac system with complex-valued integrable potential. Differ. Equ. 49, 545-556 (2013)

[21] Savchuk, A.M., Sadovnichaya, I.V.: The Riesz basis property of generalized eigenspaces for a Dirac system with integrable potential. Dokl. Math. 91, 309312 (2015)

[22] Savchuk, A.M., Sadovnichaya, I.V.: Estimates of Riesz constants for the Dirac system with an integrable potential. Differ. Equ. 54, 748-757 (2018)

[23] Savchuk, A.M., Sadovnichaya, I.V.: Spectral analysis of one-dimentional Dirac system with summable potential and Sturm-Liouville operators with distribution coefficients. Sovrem. Mat. Fundam. Napravl. 66, 373-530 (2020)

[24] Savchuk, A.M., Sadovnichaya, I.V.: Equiconvergence of spectral decompositions for Sturm-Liouville operators: triples of lebesgue spaces. Lobachevskii J. Math. 42, 1027-1052 (2021)

[25] Savchuk, A.M., Shkalikov, A.A.: Dirac operator with complex-valued summable potential. Math. Notes 96, 777-810 (2014)

[26] Zettl, A.: Sturm-Liouville theory. American Mathematical Society, Providence (2005)

Łukasz Rzepnicki $(\bowtie)$

Faculty of Mathematics and Computer Science

Nicolaus Copernicus University

ul. Chopina 12/18

87-100 Toruñ

Poland

e-mail: keleb@mat.umk.pl

Received: August 26, 2020.

Revised: August 30, 2021. 